\title{
SOLUÇÃO VIA GITT DO ESCOAMENTO MHD COM TRANSFERÊNCIA DE CALOR E MASSA DE UM BIOFLUIDO EM UM CANAL DE PLACAS PARALELAS
}

\author{
H. K. MIYAGAWA ${ }^{1}$, I. V. CURCINO ${ }^{2}$, F. A. PONTES ${ }^{1}$, P. C. PONTES ${ }^{3}$ e J. N. N. \\ QUARESMA ${ }^{2}$
}

${ }^{1}$ Universidade Federal do Pará, Programa de Pós-Graduaçao em Engenharia de Recursos Naturais da Amazônia

${ }^{2}$ Universidade Federal do Pará, Faculdade de Engenharia Química

${ }^{3}$ Universidade Federal do Sul e Sudeste do Pará, Instituto de Engenharia do Araguaia E-mail para contato: helderkm@ufpa.br

\begin{abstract}
RESUMO - Foi estudado o escoamento transiente pulsátil através de um canal poroso do tipo Darcy-Forchhmeir de um biofluido influenciado por um campo magnético externo aplicado transversalmente ao canal com reação química. $\mathrm{Na}$ formulação foi considerado o escoamento laminar, bidimensional, incompressível, eletricamente condutor, através de um meio poroso, dentro de um canal formado entre placas planas paralelas com transpiração da parede. Os campos de velocidade, concentração e temperatura foram solucionados utilizando a Técnica da Transformada Integral Generalizada e as soluções obtidas foram comparadas com as da literatura obtendo-se uma boa concordância. Foi avaliada ainda a influência do número de Prandtl no campo de temperatura, do número de Schmidt na distribuição de concentração e do número de Reynolds no perfil de velocidade, observando-se que o processo é fortemente sensível aos efeitos deste último.
\end{abstract}

\section{INTRODUÇÃO}

A fluidodinâmica de fluidos biológicos (biofluidodinâmica), como o sangue, submetidos à influência de um campo magnético externo é conhecida como BFD (Biomagnetic Fluid Dynamics). As propriedades magnéticas do sangue surgem das interações da hemoglobina, da membrana celular e da proteína intercelular (Higashi et al., 1993). O sangue ainda apresenta comportamento viscoelástico sobre certas condições em função das estruturas internas e dos eritrócitos individuais (Fukada e Kaibara, 1980; Stoltz e Lucius, 1981).

Segundo Ritman e Lerman (2007), em algumas doenças uma estrutura porosa é formada dentro das artérias por substâncias gordurosas (colesterol e coágulos sanguíneos), as quais em excesso podem levar a problemas como infarto do miocárdio ou acidente vascular cerebral, e que, portanto, demanda especial atenção no desenvolvimento de estratégias que permitam melhor compreender e, consequentemente, desenvolver medidas de remediação de tais doenças.

Em alternativa aos métodos numéricos clássicos, a técnica híbrida denominada GITT ou Técnica da Transformada Integral Generalizada (do inglês Generalized Integral Transform Technique) mostra-se capaz de tratar uma grande variedade de problemas não-lineares (Cotta e 
Mikhailov, 1997). A mesma é baseada no uso de expansões de autofunções ortogonais para expressar as variáveis dependentes desconhecidas (Sphaier et al., 2011).

Pontes et al. (2016) avaliaram o escoamento magnetohidrodinâmico oscilatório de um fluido micropolar eletricamente condutor em um meio poroso com reação química via GITT, estudando os efeitos do campo magnético e reação química de primeira ordem com coeficientes constantes, obtendo resultados em excelente concordância com os dados da literatura.

Neste contexto, o objetivo do presente trabalho consiste na solução do escoamento transiente pulsátil (modelando o fenômeno de bombeamento do coração) com transferência de calor e massa de sangue em um canal de placas paralelas (representado a geometria das artérias) através de um meio poroso do tipo Darcy-Forchhmeir (condição de algumas doenças cardíacas) e na presença de uma reação homogênea e irreversível de primeira ordem e a um campo magnético transversal constante por meio da aplicação da transformação integral. É realizada a análise de convergência das expansões em série, a avaliação dos efeitos de alguns parâmetros físicos sobre os perfis de velocidade $(U)$, temperatura $(\theta)$ e concentração $(\phi)$, os quais são apresentados graficamente, bem como é feita a verificação numérica dos resultados, comparando-se os mesmos com os resultados de Ali et al. (2015).

\section{METODOLOGIA DE SOLUÇÃO}

No presente trabalho é considerado o escoamento laminar pulsátil bidimensional transiente de um fluido biológico, incompressível, eletricamente condutor, através de um meio poroso, dentro de um canal de placas paralelas com transpiração da parede, sob a ação de um campo magnético transversal em condições não isotérmicas e com reação química. Os modelos adimensionais filtrados no domínio $-1<\eta<1, t \geq 0$ com as respectivas condições iniciais e de contorno para os campos de velocidade, temperatura e concentração foram obtidos a partir do trabalho de Ali et al. (2015), conforme é mostrado a seguir:

$$
\begin{aligned}
& \frac{\partial U}{\partial t}+\frac{\partial U}{\partial \eta}=-\frac{\partial P}{\partial \xi}+\frac{1}{\operatorname{Re}}\left(1+\frac{1}{\beta}\right) \frac{\partial^{2} U}{\partial \eta^{2}}-\left(N_{m}+\frac{1}{\lambda}\right) U-N_{f} U^{2} \\
& \frac{\partial \theta_{H}}{\partial t}+\frac{\partial \theta_{H}}{\partial \eta}+1=\frac{1}{\operatorname{Re} \operatorname{Pr}} \frac{\partial^{2} \theta_{H}}{\partial \eta^{2}}, \quad \frac{\partial \phi_{H}}{\partial t}+\frac{\partial \phi_{H}}{\partial \eta}+1=\frac{1}{S c} \frac{\partial^{2} \phi_{H}}{\partial \eta^{2}}-\frac{\gamma}{\operatorname{Re}} \phi_{H}-\frac{\gamma}{\operatorname{Re}} \eta \\
& U=0, \quad \theta_{H}=0, \quad \phi_{H}=0 \quad \text { em } \quad t=0 \\
& U=0, \quad \theta_{H}=0, \quad \phi_{H}=0 \quad \text { em } \quad \eta=-1 \\
& U=0, \quad \theta_{H}=0, \quad \phi_{H}=0 \quad \text { em } \quad \eta=1
\end{aligned}
$$

Em que as seguintes propostas de solução filtro: $\theta(\eta, t)=\theta_{H}(\eta, t)+\eta$ e $\phi(\eta, t)=\phi_{H}(\eta, t)+\eta$ foram utilizadas. O gradiente de pressão bem como os principais grupos adimensionais que foram utilizados nesta formulação são mostrados a seguir:

$$
-\frac{\partial P}{\partial \xi}=P_{s}+P_{0} \cos (\omega t) ; U=\frac{u}{v_{0}} ; \xi=\frac{x}{a} ; \eta=\frac{y}{a} ; t=\frac{v_{0}}{a} \tau ; P=\frac{P}{\rho v_{0}^{2}} ;
$$




$$
\begin{aligned}
& \theta=\frac{T-T_{m}}{T_{2}-T_{m}} ; \phi=\frac{C-C_{m}}{C_{2}-C_{m}} ; \operatorname{Re}=\frac{a v_{0}}{v_{B}} ; \lambda=\frac{k_{p} v_{0}}{a v_{B}} ; N_{f}=a b ; N_{m}=\frac{\sigma B_{0} a}{\rho v_{0}} \\
& S c=\frac{a v_{0}}{D} ; \gamma=\frac{k a^{2}}{v_{B}} ; \operatorname{Pr}=\frac{v_{B}}{\alpha}
\end{aligned}
$$

Em que Re é o número de Reynolds, $\lambda$ é o parâmetro de Darcy, $N_{f}$ é o parâmetro de arraste quadrático de Forchheimer, $N_{m}$ é o parâmetro magnético, $S c$ é o número de Schmidt, $\gamma$ é um parâmetro relacionado a reação, Pr é o número de Prandtl e $P_{s}$ e $P_{0}$ são as componentes estática e oscilatória (com frequência $\omega$ ) do gradiente de pressão.

Özisik (1993) apresenta o problema de autovalor apropriado para fundamentar a construção do potencial como uma expansão em autofunções de base ortogonal, conforme Equações 4a-c, cuja autofunção na versão normalizada é apresentada nas Equações 4d-g.

$$
\begin{aligned}
& \frac{d^{2} \psi_{i}}{d \eta^{2}}=-\mu_{i}^{2} \psi_{i} ; \quad \psi_{i}(-1)=0 ; \quad \psi_{i}(1)=0 \\
& \tilde{\psi}_{i}(\eta)=\frac{\psi_{i}(\eta)}{\sqrt{N_{i}}}=\operatorname{sen}\left[\mu_{i}(\eta+1)\right] ; \quad N_{i}=\int_{-1}^{1} \psi_{i}^{2}(\eta) \mathrm{d} \eta ; \mu_{i}=i \pi / 2, \quad i=1,2,3, \ldots
\end{aligned}
$$

Baseando-se na propriedade de ortogonalidade das autofunções é possível definir os seguintes pares transformada-inversa para os potenciais filtrados:

Transformada

$$
\begin{array}{ll}
\bar{U}_{i}(t)=\int_{-1}^{1} \tilde{\psi}_{i}(\eta) U(\eta, t) d \eta ; & U(\eta, \mathrm{t})=\sum_{i=1}^{\infty} \tilde{\psi}_{i}(\eta) \bar{U}_{i}(t) \\
\bar{\theta}_{H, i}(t)=\int_{-1}^{1} \tilde{\psi}_{i}(\eta) \theta_{H}(\eta, t) d \eta ; & \theta_{H}(\eta, \mathrm{t})=\sum_{i=1}^{\infty} \tilde{\psi}_{i}(\eta) \bar{\theta}_{H, i}(t) \\
\bar{\phi}_{H, i}(t)=\int_{-1}^{1} \tilde{\psi}_{i}(\eta) \phi_{H}(\eta, t) d \eta ; & \phi_{H}(\eta, \mathrm{t})=\sum_{i=1}^{\infty} \tilde{\psi}_{i}(\eta) \bar{\phi}_{H, i}(t)
\end{array}
$$

Inversa

A transformação integral do problema original é realizada operando-se com $\int_{-1}^{1} \tilde{\psi}_{i}(\eta) \_d \eta$ nas Equações 1a-c de modo que o seguinte sistema é obtido:

$$
\begin{aligned}
& \frac{d \bar{U}_{i}}{d t}+\sum_{j=1}^{N T} A_{i, j} \bar{U}_{j}=D_{i}(t)-\left[\frac{1}{\operatorname{Re}}\left(1+\frac{1}{\beta}\right) \mu_{i}^{2}-N_{m}-\frac{1}{\lambda}\right] \bar{U}_{i}-N_{f} \sum_{j}^{N T} \sum_{k}^{N T} F_{i, j, k} \bar{U}_{j} \bar{U}_{k} \\
& \frac{d \bar{\theta}_{H, i}}{d t}+\sum_{j=1}^{N T} A_{i, j} \bar{\theta}_{H, i}+G_{i}=-\frac{\mu_{i}^{2}}{\operatorname{Re} \operatorname{Pr}} \bar{\theta}_{H, i} \\
& \frac{d \bar{\phi}_{H, i}}{d t}+\sum_{j=1}^{N T} A_{i, j} \bar{\phi}_{H, i}+G_{i}=-\frac{\mu_{i}^{2}}{S c} \bar{\phi}_{H, i}-\frac{\gamma}{\operatorname{Re}} \bar{\phi}_{H, i}-\frac{\gamma}{\operatorname{Re}} H_{i} \\
& \bar{U}_{i}=0, \bar{\theta}_{H, i}=0, \bar{\phi}_{H, i}=0 \quad \text { em } \quad t=0
\end{aligned}
$$

Em que os coeficientes são definidos por: 


$$
\begin{aligned}
& A_{i j}=\int_{-1}^{1} \tilde{\psi}_{i} \tilde{\psi}_{j}^{\prime} d \eta ; \quad D_{i}(t)=\int_{-1}^{1} \tilde{\psi}_{i}\left[P_{s}+P_{0} \cos (\omega t)\right] d \eta ; \quad F_{i j k}=\int_{-1}^{1} \tilde{\psi}_{i} \tilde{\psi}_{j} \tilde{\psi}_{k} d \eta \\
& G_{i}=\int_{-1}^{1} \tilde{\psi}_{i} d \eta ; \quad H_{i}=\int_{-1}^{1} \tilde{\psi}_{i} \eta d \eta
\end{aligned}
$$

As Equações 6a-7e, válidas no domínio $-1<\eta<1$ e $t \geq 0$, definem um sistema de equações diferenciais ordinárias (EDOs) acoplado a ser resolvido numericamente para cada um dos potenciais transformados investigados (velocidade, temperatura e concentração) conforme é apresentado a seguir.

\section{RESULTADOS E DICUSSÃO}

A solução numérica do sistema definido nas Equações $7 \mathrm{a}-\mathrm{c}$ foi implementada na plataforma de computação numérico-simbólica Mathematica 9.0 (Wolfram, 2005) em modo default utilizando a função NDSOLVE para NT (número de termos) suficientemente grande de modo a fornecer uma aproximação satisfatória da expansão em séries.

A análise de convergência das soluções via GITT foi calculada em algumas posições ao longo de $\eta$ para $t=0,3$. A Tabela 1 mostra que o campo de velocidade apresenta quatro dígitos completamente convergidos com 40 termos enquanto que 30 termos são necessários para assegurar uma solução numérica com quatro dígitos completamente convergidos para os campos de temperatura e de concentração.

Tabela 1 - Análise de Convergência para os resultados obtidos pela GITT com $\operatorname{Pr}=5, S c=0,7$,

\begin{tabular}{c|ccccccc}
\multirow{7}{*}{$\operatorname{Re}=1, N_{m}=0,3, N_{f}=0,002, \omega=8, P_{s}=10, P_{0}=7, \lambda=5, \beta=4$ e $\gamma=4$} \\
\hline
\end{tabular}

Os resultados obtidos para o campo de velocidade, temperatura e concentração foram verificados numericamente com os obtidos por Ali et al. (2015) mostrando boa concordância entre os resultados, o que pode ser evidenciado nas Figuras 1a-c, que ilustram também a influência de alguns parâmetros nos campos de velocidade, temperatura e concentração. 
Conforme a Figura 1a, mantidos constantes os demais parâmetros o efeito do aumento de Re é acelerar o fluido através do canal, uma vez que para $\operatorname{Re}=1,5$ as forças de inércia são de magnitude maior do que as forças viscosas, ao contrário do que acontece em $\operatorname{Re}=0,2$. Porém, as propriedades não uniformes do fluido proporcionam um aumento na velocidade de sucção na placa superior (posicionada em $\eta=1$ ), deslocando os perfis para a parede superior do canal.

A Figura $1 \mathrm{~b}$ mostra que o aumento dos valores de Pr influencia significativamente o perfil de temperatura perto das paredes do canal, mesmo levando em conta que para $\operatorname{Pr}=5$ a taxa de difusão térmica é de menor magnitude do que a taxa de difusão viscosa, quando comparado com o caso em que $\operatorname{Pr}=25$.

Na Figura 1c observa-se que o número de Sc não influencia os perfis de concentração próximos à parede inferior do canal enquanto que no restante do canal observa-se a diminuição do valor da concentração. Assim, para $S c=0,15$ a taxa de difusão mássica é de magnitude muito maior do que taxa de difusão viscosa, quando comparado com o caso em que $S c=1$.

Figura 1 - Influência do (a) número de Reynolds no campo de velocidade, (b) número de Prandtl no campo de temperatura e (c) número de Schmidt no campo de concentração com
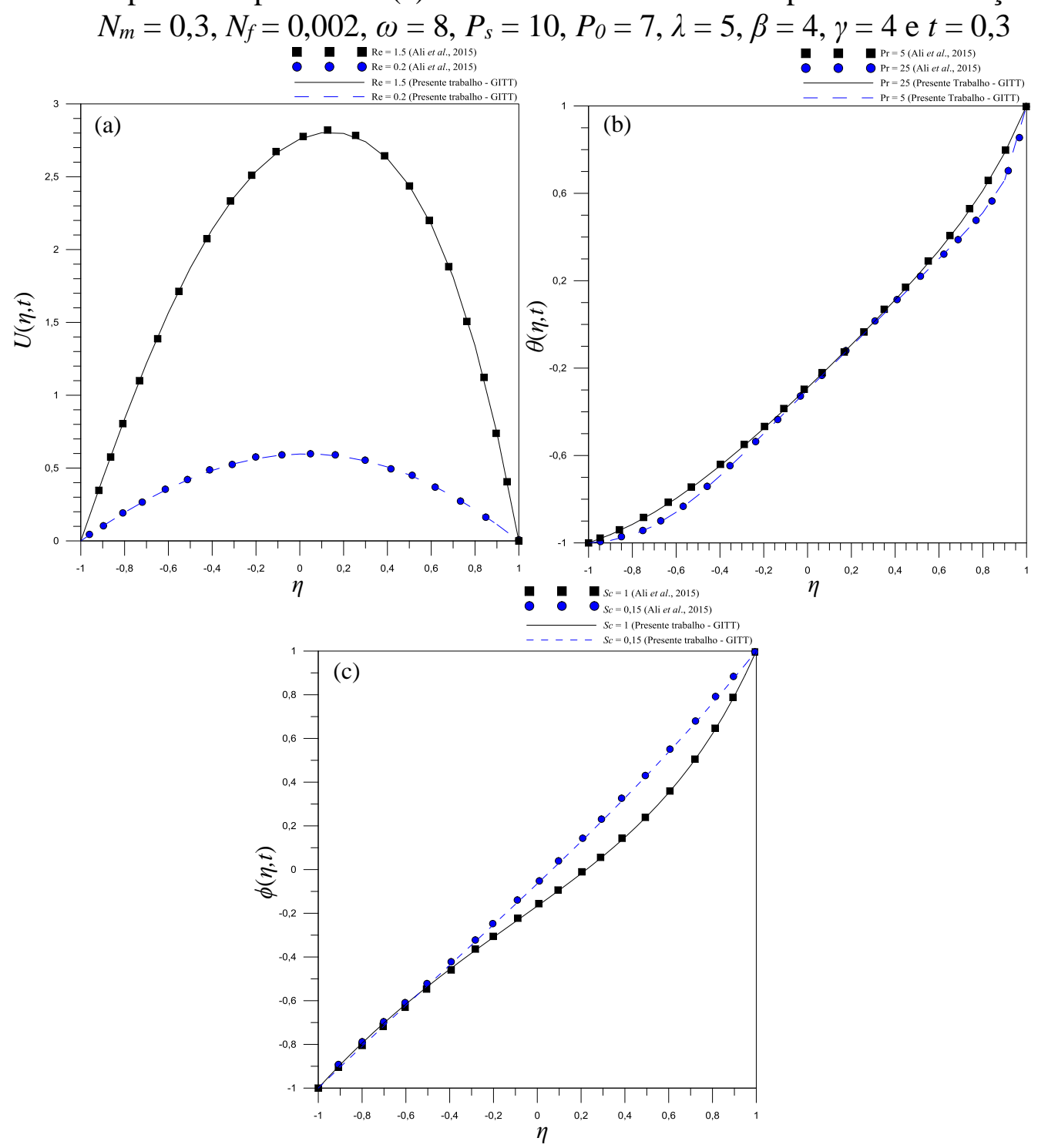


\section{CONCLUSÃO}

Um modelo não-linear para o escoamento biofluidodinâmico do sangue através de um meio poroso, dentro de um canal de placas paralelas com transpiração da parede, sob a ação de um campo magnético transversal foi solucionado pela GITT. A convergência das soluções foi realizada e os resultados da GITT foram satisfatoriamente verificados com a literatura.

A técnica apresentou-se como um método robusto e versátil na solução desta classe de problemas que envolvem fenômenos eletromagnéticos com reação química, permitindo a obtenção de resultados em excelente concordância com dados da literatura e possibilitando o desenvolvimento de soluções de problemas mais complexos.

O aumento do número de Reynolds é capaz de acelerar o escoamento no canal, enquanto que o aumento no número de Prandtl causa efeitos ao campo de temperatura de forma mais pronunciada próximo as paredes do canal. Já o aumento do número de Schmidt proporciona uma diminuição da concentração apenas na região próxima a parede superior do canal.

\section{REFERÊNCIAS}

ALI, K.; AHMAD, S.; ASHRAF, M. Numerical simulation of MHD pulsatile flow of a biofluid in a channel. AIP Advances, v.5, p. 087130, 2015.

COTTA, R. M.; MIKHAILOV, M.D. Heat conduction: lumped analysis, Integral transforms, Symbolic Computation. John Wiley \& Sons, 1997.

FUKADA, E.; KAIBARA, M. Biorheology, v.17, p.177, 1980.

HIGASHI, T.; YAMAGISHI, A.; TAKEUCHI, T.; KAWAGUCHI, N.; SAGAWA, S.; ONISHI, S.; DATE, M. Blood, v.82, p.1328, 1993.

ÖZISIK, M. N. Heat Conduction, John Wiley \& Sons, New York, 1993.

PONTES, F. A; MIYAGAWA, H. K.; CURCINO, I. V.; PONTES, P. C.; MACÊDO, E. N.; QUARESMA, J. N. N.; Transformação Integral do Escoamento Magnetohidrodinâmico Oscilatório de um Fluido Micropolar Eletricamente Condutor em um Meio Poroso Com Reação Química. In: Anais do XXI Congresso Brasileiro de Engenharia Química COBEQ 2016. Fortaleza-CE, 2016.

RITMAN, E.L. LERMAN, A. Current Cardiology Reviews. 3, 43, 2007.

SHARMA, B.R. BORGOHAIN, D. International Journal of Innovative Research in Science, Engineering and Technology. 7, 14867, 2014.

SPHAIER, L. A.; COTTA, R. M.; NAVEIRA-COTTA, C. P.; QUARESMA, J. N. N. The UNIT algorithm for solving one-dimensional convection-diffusion problems via integral transforms. I. C. Heat and Mass Transfer. v. 38, p. 565-571, 2011.

STOLTZ, J. F.; LUCIUS, M. Biorheology, v.18, p.453, 1981.

WOLFRAM, S. MATHEMATICA - a system for doing mathematics by computer, in: The Advanced Book Program. Addison Wesley, Reading, MA, 2005. 